\title{
ANALYSIS OF LAY-UP OPTIMALITY CONDITIONS FOR BUCKLING OPTIMIZATION OF VAT (STEERED FIBER) COMPOSITE PLATES
}

\author{
Sergey Selyugin \\ AIRBUS Operations GmbH, Kreetslag 10, 21129 Hamburg, Germany \\ e-mail: sergey.selyugin@airbus.com
}

\begin{abstract}
In the present paper the analysis of the lay-up optimality conditions (derived earlier) for buckling optimization of VAT composite plates is performed. The plates have a symmetric lay-up and loaded along their contour by the in-plane forces. The consideration employs the von Karman approach. The lay-up optimality conditions correspond to the maximization of a single-mode (lowest) buckling eigenvalue. The conditions are comprehensively analyzed. The conclusions of the analysis are presented. The key conclusion is that the optimality conditions with and without account of variation of the in-plane forces are the same.
\end{abstract}

Keywords: composite plate, buckling, lay-up, steered fibers, optimality

(C) Sergey Selyugin, 2020 


\section{Introduction}

The buckling optimality of composite plates is studied in the last two-three decades by many authors (see, e.g., the reviews of Ghiasi et al. 2009, 2010 and the book of Falzon and Aliabadi 2008). The works in the field consider the problem mainly from the point of view of numerical solutions.

In the paper of Selyugin (2019) the lay-up optimality conditions for buckling optimization of the VAT (steered fiber) composite plates have been derived. The present paper is devoted to the theoretical analysis of the conditions.

Section 2 contains some theoretical background.

In Section 3 the analysis of the optimality conditions is performed.

Section 4 contains the conclusions.

\section{Theoretical background}

We consider the laminated composite plate with the symmetric lay-up. The lay-up is composed of the orthotropic fiber-reinforced plies of the same thickness $h_{p l y}$ and various pointwise orientation angles. The total number of plies is even and equal to $2 K$, the generalization to the odd ply number is straightforward and is not considered in the present paper. Fig. 1 illustrate the plate and the notations. 


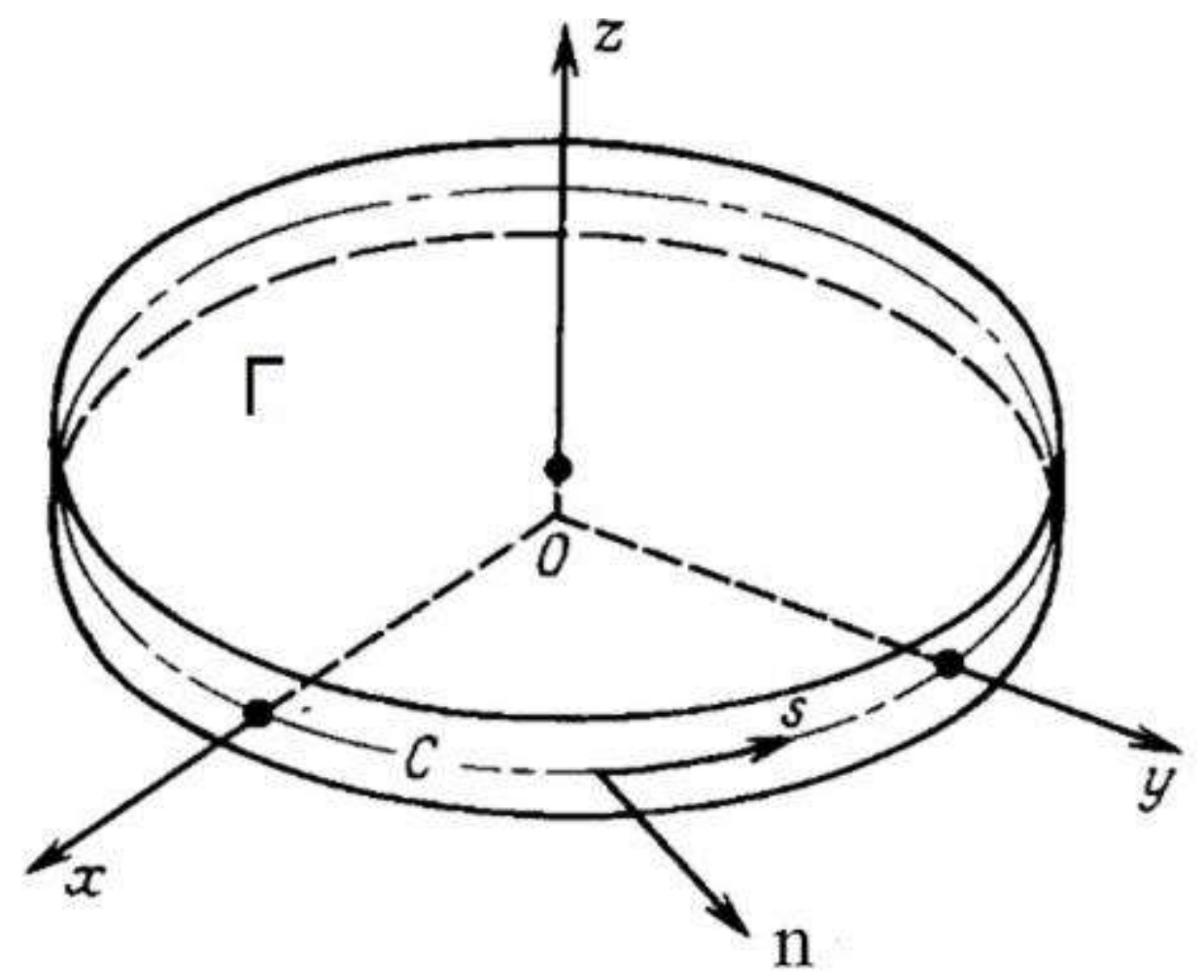

Fig. 1

The mid-plane of the flat plate is located in the $X Y$ plane. The plate mid-plane is restricted by the piecewise smooth contour $C$ with external normal $n$ (having the components $m, l$ ) and tangent direction $s$; the directions $n, l$ and $\mathrm{Z}$ create a right-hand triplet. The coordinate system $X Y Z$ is a Cartesian one.

The plate is loaded by the in-plane forces, acting at the part $C_{1}$ of the contour $C$. The remaining part $C_{2}$ of the contour the plate is not moving in $X$ and $Y$. The plate is simply supported, i.e., the $z$-displacement and the normal bending moment $M_{v}$ acting perpendicular to the contour $C$ are equal to zero. The clamping boundary conditions are not considered in the present paper, the change to the case is straightforward. 
The $X, Y, Z$ displacements are denoted as $u, v, w$, respectively.

The signs of the loads are the following. The force flows $N_{x}, N_{y}$ (along $X$ and along $Y$ ) are positive in tension, the shear force flow $N_{x y}$ is positive when it decreases the $90^{\circ}$ angle between the orthogonal lines at the plate surface. The flows are in equilibrium. The Composite Lamination Plate Theory (CLPT) is used for description of plate deflections (see the book of Gibson 1994).

The Classical Lamination Plate Theory (CLPT), based on linear-elastic relations for every layer and Bernoulli's hypotheses, is used for obtaining the equation for the deflections $w$ (see the book of Gibson 1994). The equation is written as follows:

$$
\begin{aligned}
& D_{11} \frac{\partial^{4} w}{\partial x^{4}}+2\left(D_{12}+2 D_{66}\right) \frac{\partial^{4} w}{\partial x^{2} \partial y^{2}}+D_{22} \frac{\partial^{4} w}{\partial y^{4}}+4 D_{16} \frac{\partial^{4} w}{\partial x^{3} \partial y}+4 D_{26} \frac{\partial^{4} w}{\partial x \partial y^{3}}- \\
& -N_{x} \frac{\partial^{2} w}{\partial x^{2}}-N_{y} \frac{\partial^{2} w}{\partial y^{2}}-2 N_{x y} \frac{\partial^{2} w}{\partial x \partial y}=0
\end{aligned}
$$

where $D_{i j}, i=1,2,6 ; j=1,2,6$, are the elements of the bending stiffness matrix $D$, coupling the bending/twisting moments and various second derivatives of the deflection $w$ with respect to $x$ and $y$. The coupling is written as follows:

$$
\left[\begin{array}{l}
M_{x} \\
M_{y} \\
M_{x y}
\end{array}\right]=\left[\begin{array}{lll}
D_{11} & D_{12} & D_{16} \\
D_{12} & D_{22} & D_{26} \\
D_{16} & D_{26} & D_{66}
\end{array}\right]\left[\begin{array}{c}
-\frac{\partial^{2} w}{\partial x^{2}} \\
-\frac{\partial^{2} w}{\partial y^{2}} \\
-2 \frac{\partial^{2} w}{\partial x \partial y}
\end{array}\right]
$$


We denote the left-hand side of (2) as a vector-column $\vec{M}$, and as $\vec{k}$ the vector-column at the right-hand side (which is multiplied to $D$ matrix). Also we use the notations for the corresponding curvatures:

$$
k_{x}=-\frac{\partial^{2} w}{\partial x^{2}} ; k_{y}=-\frac{\partial^{2} w}{\partial y^{2}} ; k_{x y}=-\frac{\partial^{2} w}{\partial x \partial y}
$$

Then (2) is rewritten in the matrix-vector form as:

$$
\vec{M}=D \vec{k}
$$

With the boundary conditions indicated above, the boundary-value problem for deflections is completed.

The distribution of the in-plane stress flows is given by the relations:

$$
\vec{N}=A\left[\begin{array}{c}
u_{, x} \\
v_{, y} \\
u_{, y}+v_{, x}
\end{array}\right]
$$

where $A$ is the in-plane stiffness matrix, and the column-vector

$$
\vec{N}=\left(N_{x}, N_{y}, N_{x y}\right)^{T}
$$

the superscript $T$ means transposing, and index after comma means the differentiation w.r.t. the corresponding coordinate.

The components of the $\vec{N}$ must satisfy the equilibrium equations, and the in-plane displacements must satisfy the corresponding boundary conditions. 
It is supposed that the buckling eigenvalue is a single-modal one. The optimization goal is to design a lay-up maximizing the first (lowest) eigenvalue.

As it is shown in Selyugin (2019), the first order necessary local optimality conditions for the ply orientation angle $\theta_{i}$ are written as follows, $i, \ldots, K$ :

$$
\sin 2\left(\theta_{i}-\psi\right)\left[\frac{U_{2}}{4 U_{3}}\left(k_{1}^{2}-k_{2}^{2}\right)+\left(k_{1}-k_{2}\right)^{2} \cos 2\left(\theta_{i}-\psi\right)\right] \frac{1}{6}\left(z_{i}^{3}-z_{i-1}^{3}\right)=0, i=1, \ldots, K
$$

where all angles are measured relatively to the $X$ axis, $k_{1}, k_{2}, \psi, z_{i}, z_{i-1}$ respectively are the largest and the lowest principal curvatures, the angle between the direction $k_{1}$ and the $\mathrm{X}$ axis, the top and the bottom $z$ coordinates of the $i$-th layer, $i=1, \ldots, K$. The value of $z_{0}$ is equal to zero. The relation (7) was obtained in the more simplified case in (Selyugin, 2013) using (Gibson, 1994) background on composites.

\section{Analysis of the optimality conditions}

The first conclusion coming from (Selyugin, 2019) is that the lay-up optimality conditions in the case of varied in-plane forces of the plate are the same as (7). This means that the in-plane force variation doesn't influence the optimality conditions.

It may be also noticed from the optimality condition derivation (Selyugin, 2019) that the parameters $\alpha_{1}, \alpha_{2}$ there (they are some Lagrange multipliers) are some generalized in-plane displacements.

Further we look at the typical deflection buckling shape for the 2D uniformly compressed simply supported composite plate shown in Figure 2. The Figure will support us in our analysis. 


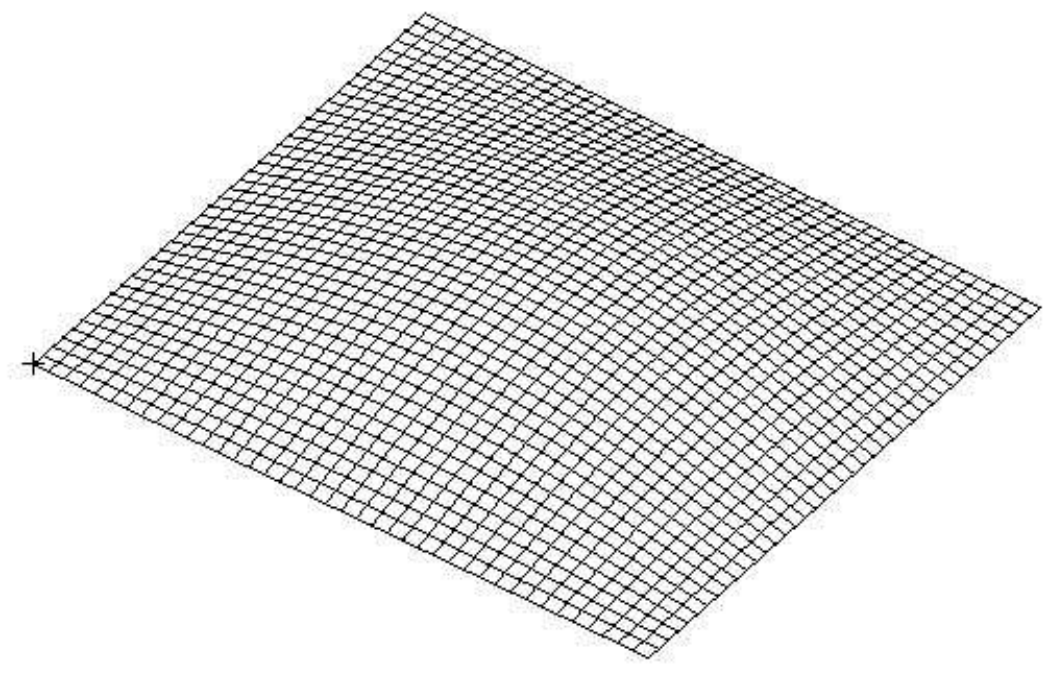

Fig. 2. Typical buckling shape of the $2 \mathrm{D}$ compressed composite plate.

Now we analyze the relation (7) in details. Rewrite (7) without the mutual multiplier as

$$
\sin 2\left(\theta_{i}-\psi\right)\left[\frac{U_{2}}{4 U_{3}}\left(k_{1}^{2}-k_{2}^{2}\right)+\left(k_{1}-k_{2}\right)^{2} \cos 2\left(\theta_{i}-\psi\right)\right]=0, i=1, \ldots, K
$$

The umbilical points (where $k_{1}=k_{2}$ ) are not discussed below.

First we consider the term within the square brackets. When it is equal to zero, we have

$$
\cos 2\left(\theta_{i}-\psi\right)=-\frac{U_{2}\left(k_{1}+k_{2}\right)}{4 U_{3}\left(k_{1}-k_{2}\right)}
$$

We remember that the value of the ratio $\frac{U_{2}}{4 U_{3}}$ is about 1.0 (see Selyugin, 2013). When the both principal curvatures $k_{1}, k_{2}$ are of the same sign (see Fig. 1) and rather close to each other in values (e.g., 5.0 and 4.0), there is no solution $\theta_{i}$ of (9). But in principle, for some combination of the principal curvature values, there are some solutions of (9). 
The equation

$$
\sin 2\left(\theta_{i}-\psi\right)=0
$$

gives the solutions

$$
\theta_{i}=\psi \quad \text { or } \quad \theta_{i}=\psi \pm \pi / 2
$$

The solutions mean that the ply material direction coincide the first or the second principal curvature direction.

It was shown in (Selyugin, 2013) that the sum of (8) for $i=1, \ldots, K$ leads to a relation

$$
M_{x y}^{\text {pr.cur. }}\left(k_{1}-k_{2}\right)=0
$$

where $M_{x y}^{p r . c u r .}$ is the twisting moment measured in the principal curvature axes. The relation (12) means that the moment tensor and the tensor of curvatures are co-axial. In fact, the optimal plate is a locally orthotropic plate.

\section{Conclusions}

- Possible solutions of the optimality conditions are discussed;

- The variation of the in-plane forces does not influence the optimality conditions obtained without account of such variation;

- The plate with optimal lay-up is a locally orthotropic one.

\section{Acknowledgement}

The author is thankful to the colleagues for the fruitful discussions. 


\section{REFERENCES}

Falzon BG, Aliabadi MH Buckling and postbuckling structures: experimental, analytical and numerical studies, Imperial College Press, 2008.

Ghiasi H, Pasini D, Lessard L 2009. Optimum Stacking Sequence Design of Composite Materials Part I: Constant Stiffness Design. Compos Struct 90: 1-11.

Ghiasi H, Fayazbakhsh K, Pasini D, Lessard L 2010. Optimum Stacking Sequence Design of Composite Materials Part II: Variable Stiffness Design. Compos Struct 93: 1-13

Gibson RF 1994. Principles of composite material mechanics. McGraw-Hill, Inc., 425 pp.

Kassapoglou C 2010. Design and Analysis of Composite Structures: With Applications to Aerospace Structures. Wiley.

Selyugin S 2013. On Choice of Optimal Anisotropy of Composite Plates against Buckling, with Special Attention to Bending-twisting Coupling. Struct Multidisc Optim 48: 279-294 
Selyugin S 2019. Lay-up optimality conditions for buckling level maximization of VAT (steered fiber) composite plates. Preprint https://www.researchgate.net/publication/337137413 LAYUP_OPTIMALITY_CONDITIONS_FOR_BUCKLING_LEVEL_MAXIMIZATION_OF_VAT STEERED_FIBER_COMPOSITE_PLATES 